\title{
Cervical Spinal Cord DTI Is Improved by Reduced FOV with Specific Balance between the Number of Diffusion Gradient Directions and Averages
}

\author{
(D) A. Crombe, (D) N. Alberti, (DB. Hiba, (D) M. Uettwiller, (D) V. Dousset, and DT. Tourdias
}

\begin{abstract}
BACKGROUND AND PURPOSE: Reduced-FOV DTI is promising for exploring the cervical spinal cord, but the optimal set of parameters needs to be clarified. We hypothesized that the number of excitations should be favored over the number of diffusion gradient directions regarding the strong orientation of the cord in a single rostrocaudal axis.
\end{abstract}

MATERIALS AND METHODS: Fifteen healthy individuals underwent cervical spinal cord MR imaging at 3T, including an anatomic 3DMulti-Echo Recombined Gradient Echo, high-resolution full-FOV DTI with a NEX of 3 and 20 diffusion gradient directions and 5 sets of reduced-FOV DTIs differently balanced in terms of NEX/number of diffusion gradient directions: (NEX/number of diffusion gradient directions $=3 / 20,5 / 16,7 / 12,9 / 9$, and 12/6). Each DTI sequence lasted 4 minutes 30 seconds, an acceptable duration, to cover Cl-C4 in the axial plane. Fractional anisotropy maps and tractograms were reconstructed. Qualitatively, 2 radiologists rated the DTI sets blinded to the sequence. Quantitatively, we compared distortions, SNR, variance of fractional anisotropy values, and numbers of detected fibers.

RESULTS: Qualitatively, reduced-FOV DTI sequences with a NEX of $\geq 5$ were significantly better rated than the full-FOV DTI and the reduced-FOV DTI with low NEX (N=3) and a high number of diffusion gradient directions $(D=20)$. Quantitatively, the best trade-off was reached by the reduced-FOV DTI with a NEX of 9 and 9 diffusion gradient directions, which provided significantly fewer artifacts, higher SNR on trace at $b=750 \mathrm{~s} / \mathrm{mm}^{2}$ and an increased number of fibers tracked while maintaining similar fractional anisotropy values and dispersion.

CONCLUSIONS: Optimized reduced-FOV DTI improves spinal cord imaging. The best compromise was obtained with a NEX of 9 and 9 diffusion gradient directions, which emphasizes the need for increasing the NEX at the expense of the number of diffusion gradient directions for spinal cord DTI contrary to brain DTI.

ABBREVIATIONS: $\mathrm{CSC}=$ cervical spinal cord; $\mathrm{FA}=$ fractional anisotropy; $\mathrm{f}-\mathrm{FOV}=$ full-FOV; $\mathrm{MERGE}=$ Multi-Echo Recombined Gradient Echo; $\mathrm{NDGD}=$ number of diffusion gradient directions; $r$-FOV $=$ reduced-FOV

C ervical spinal cord (CSC) assessment in daily clinical routines still essentially relies on a qualitative evaluation of conventional MR imaging sequences. Nonetheless, additional sequences could provide more sensitive information about CSC alterations,

Received February 23, 2016; accepted after revision April 25.

From the Centre Hospitalier Universitaire de Bordeaux (A.C., V.D., T.T.), Service de Neurolmagerie Diagnostique de Thérapeutique, Bordeaux, France; Institut National de la Santé et de la Recherche Médicale U1215 (A.C., V.D., T.T.), Physiopathologie de la Plasticité Neuronale, Bordeaux, France; Institut de Bio-Imagerie de Bordeaux (A.C., N.A., B.H., V.D., T.T.), Université de Bordeaux, Bordeaux, France; Centre de Résonance Magnétique des Systèmes Biologiques (N.A., B.H.), Centre National de la Recherche Scientifique Unité Mixte de Recherche 5536, Bordeaux, France; Institut de Neurosciences Cognitives et Intégratives d'Aquitaine (B.H.), Centre National de la Recherche Scientifique Unité Mixte de Recherche 5287, Bordeaux, France; and GE Healthcare (M.U.), Vélizy-Villacoublay, France.

The work was supported by public grants from the French Agence Nationale de la Recherche within the context of the Investments for the Future program referenced ANR-10-LABX-57 named TRAIL (Translational Research and Advanced Imaging Laboratory) and ANR-10-LABX-43 named BRAIN (Bordeaux Region Aquitaine Initiative for Neurosciences). notably in inflammatory, traumatic, and neurodegenerative diseases. ${ }^{1-5}$ Among the sequences, diffusion tensor imaging, classically based on single-shot echo-planar imaging, could provide valuable qualitative information and quantitative surrogate biomarkers.

While DTI has been widely investigated in the brain, its use for CSC imaging is still challenging and is restricted to preclinical and monocentric clinical studies. Several reasons for these limitations include the CSC being a small structure, prone to distortions;

Paper previously presented in part at: Annual Meeting of the French Society of Radiology, October 19, 2014; Paris, France; and Annual Meeting of the French Society of Neuroradiology, April 8, 2015; Paris, France.

Please address correspondence to Amandine Crombe, Service de Neuroimagerie Diagnostique et Thérapeutique, Hopital Pellegrin, $\mathrm{CHU}$ de Bordeaux, Place Amélie Raba-Leon, 33076 Bordeaux cedex, France; e-mail: amandine.crombe@ens-lyon.fr - Indicates open access to non-subscribers at www.ajnr.org

http://dx.doi.org/10.3174/ajnr.A4850 
susceptibility artifacts, especially with a long echo-planar readout train; flow artifacts; and physiologic motion artifacts.

Among the various methods that have been implemented on MR imaging systems to improve DTI quality, reduced field-ofview (r-FOV) is particularly promising. It consists of reducing the FOV in the phase- or frequency-encoding direction to shorten the echo-planar readout train and attenuate susceptibility- and motion-related artifacts. ${ }^{6-11}$ Because the CSC is a small longitudinal structure, $\mathrm{r}-\mathrm{FOV}$ is especially suitable for its exploration. One of the r-FOV techniques uses a 2D echo-planar radiofrequency pulse to excite a rectangular FOV, with contiguous multisections. The $k$-space is then more rapidly acquired for the same spatial resolution, which decreases single-shot EPI artifacts together with inherent fat suppression. ${ }^{6}$

On qualitative analysis, this CSC r-FOV diffusion-weighted imaging method obtained from 3 orthogonal diffusion gradient directions has already demonstrated its ability to better detect anatomic details, with fewer ghosting and blurring artifacts, ${ }^{12}$ while apparent diffusion coefficient values were stable compared with conventional full-FOV CSC DWI (f-FOV).

However, there is no recommendation regarding the optimal set of parameters to perform r-FOV DTI in CSC. Some consensus has been reached (regarding the b-value, voxel size, number of excitations, number of diffusion gradient directions [NDGD], and bandwidth ${ }^{13}$ ), but these recommendations concern f-FOV DTI with parallel imaging, whose sequence scheme is different from the r-FOV scheme. The smaller voxel size in r-FOV inherently leads to a decrease of the signal-to-noise ratio. Classic strategies to recover SNR consist of increasing the NEX and/or the NDGD. Unlike brain DTI, for which the NDGD should be preferred over the NEX, there is no consensus about the best pair of NEX/NDGD for CSC DTI to handle with the complex fiber orientation. One might even hypothesize that the best trade-off of NEX/NDGD could be obtained by favoring NEX (unlike for brain DTI) because the CSC is particularly affected by artifacts and presents a single prominent orientation. It is difficult to rely on phantom studies or theoretic analyses for such optimization in the CSC, which could not take into account CSF pulsations, heartbeats, or patient respiratory motions.

Therefore, the purpose of this study was to compare different sets of r-FOV CSC DTI, depending on different pairs of NEX and NDGD, and a standard f-FOV DTI. To do so, we used an empiric nonsequential optimization approach, in which both NEX and NDGD were modified simultaneously, with the only constraint being maintaining the same clinically acceptable scan duration. To evaluate image quality, we proposed a practical clinical approach with a standardized bench test for qualitative and quantitative evaluations derived from ROIs and tractography.

\section{MATERIALS AND METHODS Patient Population}

Fifteen healthy volunteers (6 women, 9 men; age range, 22-30 years) were prospectively included at Bordeaux University Hospital after written informed consent was obtained. This study was approved by the local ethics committee review board.

\section{Imaging Methods}

All scans were obtained on a 3T MR imaging scanner (Discovery MR750W; GE Healthcare, Milwaukee, Wisconsin) equipped with high-performance gradients (maximum slew rate of $200 \mathrm{mT} /$ $\mathrm{m} / \mathrm{ms}$ and maximum strength of $44 \mathrm{mT} / \mathrm{m}$ ) and a dedicated posterior coil with 40 elements and a 19-channel phased array headneck spine coil.

Subjects were asked to breathe normally and not to swallow or cough during the acquisition. They were positioned with a slight flexion of the head and with well immobilized to reduce involuntary movements.

All sequences were performed in the axial plane with an anteroposterior phase-encoding direction and used similar geometry to perform fair comparisons. Pulse triggering was not used, to limit the scan time.

Conventional Imaging. The protocol included an anatomic sagittal T2-weighted spin-echo and a 3D Multi-Echo Recombined Gradient Echo sequence (MERGE; GE Healthcare) acquired in the same axial plane as the DTI sequences (ie, perpendicular to the long axis of the upper cervical spine). 3D-MERGE provides good white and gray matter differentiation with good SNR and was considered the anatomic reference. One hundred eight sections with a resolution of $0.8 \times 0.8 \times 0.8 \mathrm{~mm}^{3}$ covering a $140 \times 140$ $\mathrm{mm}^{2}$ FOV were acquired in 8 minutes. Other sequence parameters were the following: TR/TE, 10/5 ms; flip angle, $7^{\circ}$; turbo factor, 36 .

f-FOV DTI. Conventional CSC DTI corresponded to the DTI usually performed at our institutions, ${ }^{14}$ which was optimized beforehand according to the literature. ${ }^{15,16}$ Parallel imaging was used (array spatial sensitivity encoding technique) with an acceleration factor of 2. The single-shot EPI parameters were the following: NDGD, 20 directions; $b=0 \mathrm{~s} / \mathrm{mm}^{2}$ and $b=750 \mathrm{~s} / \mathrm{mm}^{2}$; matrix, $96 \times 96$; FOV, $120 \times 120 \mathrm{~mm}^{2}$; in-plane resolution, $1.25 \times 1.25 \mathrm{~mm}^{2}$; section thickness, $5 \mathrm{~mm}$; TR/TE, 5600/75 ms; and NEX, 3. This sequence covered the CSC from C1 to C7 within 6 minutes 50 seconds.

r-FOV DTI. For each subject, r-FOV DTI was acquired by using 5 schemes with different combinations of NDGD and NEX for each direction, starting from a sequence with many directions $(\mathrm{NDGD}=20)$ and few excitations $(\mathrm{NEX}=3)$ up to an opposite scenario obtained with the minimum number of directions to calculate a diffusion tensor (NDGD $=6$ ) and a maximum number of excitations (NEX $=12$ ). Each scheme lasted about 4 minutes 30 seconds, to cover the upper cervical spine from C1 to C4. The 5 schemes of the single-shot EPI sequence were as follows in terms of NEX/NDGD: 3N/20D, 5N/16D, 7N/12D, 9N/9D, 12N/ $6 \mathrm{D}$. The directions were noncollinear and generated according to the recommendations of Jones et $\mathrm{al}^{17}$ for the optimization of gradient vector orientations (TR/TE, 3600/75 ms). The other parameters were fixed and corresponded to those applied for the f-FOV sequence to compare r-FOV and f-FOV, notably regarding voxel size $\left(r-F O V\right.$ was $96 \times 48$ with a matrix of $120 \times 60 \mathrm{~mm}^{2}$; in-plane resolution was $1.25 \times 1.25 \mathrm{~mm}^{2}$, with a 5 -mm section thickness).

The entire protocol was performed during the same session and lasted 40 minutes. 


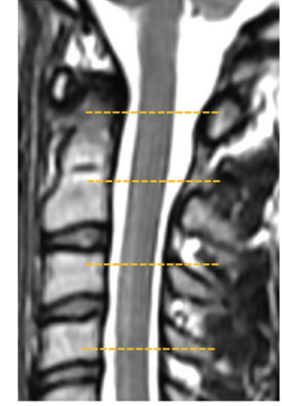

A Sagittal T2 SE

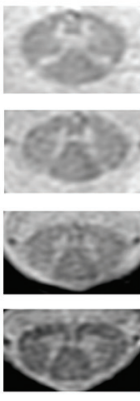

3D MERGE
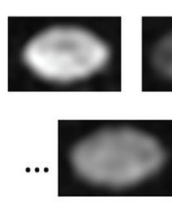

$\cdots$

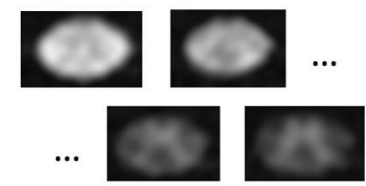

Raw DTI data
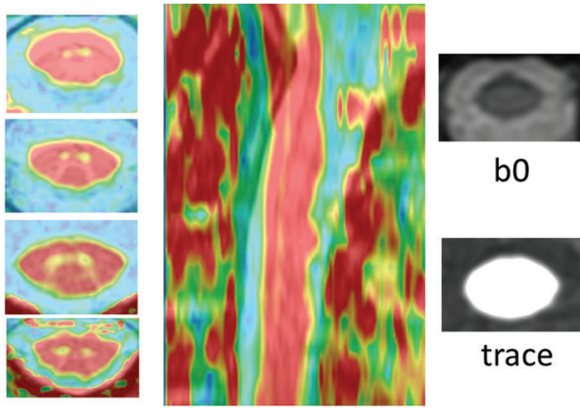

b0

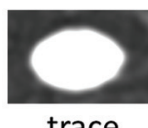

trace

B. 1
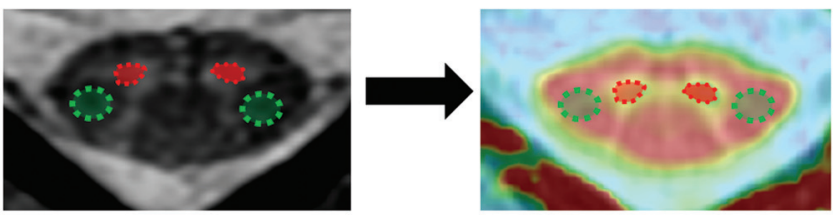

Axial Sagittal reformation

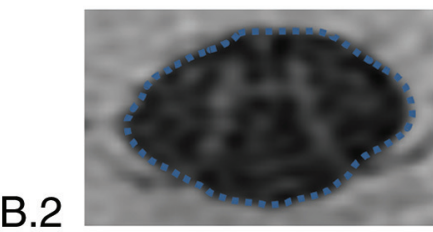

3D MERGE

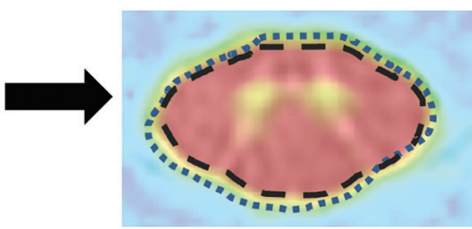

FA map

FIG 1. Postprocessing pipeline and analyses. $A$, Four sections were analyzed in detail: $\mathrm{Cl}, \mathrm{C2}, \mathrm{C} 3$, and $\mathrm{C}$. Raw data diffusion-weighted images were treated for eddy current correction before generation of DTI parameters. We focused on FA because this parameter is the most commonly used. Fusions of FA and 3D-MERGE were created at these 4 levels as well as reconstructed on the sagittal orientation to facilitate the identification of distortions and pixel misregistration. $B$, ROI positioning: ROIs (BT) were manually delineated on 3D-MERGE, on the right and left anterior horns of the cord (red area) for gray matter, and on the right and left corticospinal tract (green area) for white matter, and then propagated on the coregistered FA map. B2, If needed, ROIs were manually adjusted to account for FA map distortion. Furthermore, because of the partial volume effect at the interface between CSF and the FA map, ROls of the full section (blue dotted line, whose surface corresponded to $\mathrm{S}_{\text {[Full section - Merge] }}$ ) were adjusted to remove the pixels subject to artifacts at the periphery of the ROI (black dashed line, whose area corresponded to $\left.S_{[\text {Full Section - FA] }}\right)$.

Postprocessing. Because our goal was to identify the best set of parameters for a DTI sequence used for clinical applications, we postprocessed the images with the tools that are accessible on clinical systems. Therefore, the DTI dataset processing and the qualitative and ROI-based analyses were performed on an AW server Workstation 5 (GE Healthcare). Tractography-based analyses were performed with Olea Sphere software (Olea Medical, La Ciotat, France).

All the raw images were corrected for motion artifacts and eddy current distortion with the algorithm implemented on the DTI analysis software (GE Healthcare).

\section{Radiologic Assessment}

Qualitative Analysis. Two radiologists (A.C. and N.A., with, respectively, 4 and 7 years of experience in MR imaging) randomly evaluated the quality of each of the 6 DTI sequences (f-FOV, rFOV 3N/20D, r-FOV 5N/16D, r-FOV 7N/12D, r-FOV 9N/9D, r-FOV $12 \mathrm{~N} / 6 \mathrm{D}$ ) blinded to the nature of the sequence. They determined a score based on a 4-point scale, adapted from Zaharchuk et al ${ }^{12}$ as follows: $1=$ nondiagnostic, $2=$ poor, $3=$ moderate, 4 good. This score was based on anatomic details, artifacts, distortion, and perceived SNR from fractional anisotropy (FA) maps alone and fused with 3D-MERGE. In case of disagreement between the 2 radiologists, consensus was obtained after discussion with a third experienced neuroradiologist (T.T., with 14 years of experience).

Number of Sections with Artifacts. The number and the types of artifacts or nondiagnostic sections for each DTI sequence (flow artifacts, motion artifacts, susceptibility artifacts, major distortion) were reported and expressed as the percentage of sections with artifacts of the total number of sections.

Quantitative Comparisons of FA Values Based on ROI Analysis. For this part of the study, 1 neuroradiologist (A.C.), still blinded to the sequence, manually delineated ROIs on 4 sections passing through the middle of the vertebral bodies C1, C2, C3, and C4. ROIs were positioned twice with good reproducibility (intraclass correlation coefficient, 0.788 ; 95\% CI, 0.353-0.943).

Measurements were obtained on the following areas: 1) gray matter: left and right anterior horn of the cord (GM);2) white matter: left and right corticospinal tract (WM); and 3) a full section of the cervical spinal cord (Fig 1). ROIs were initially placed on the axial 3D-MERGE image and then propagated on coregistered FA maps. If needed, ROIs were slightly manually adjusted to account for FA map distortion. Because of partial volume effects at the interface between the CSF and spinal cord with about 2 pixels showing intermediate FA values, measurements on the full section of the spinal cord were 


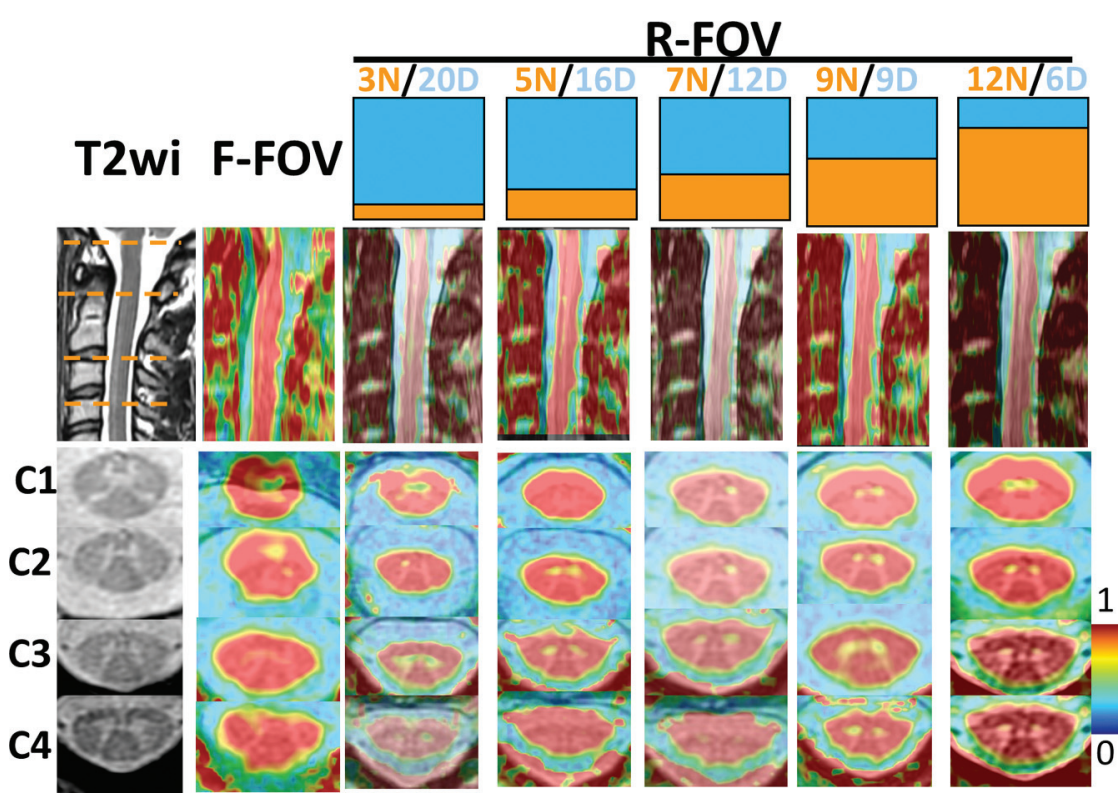

FIG 2. Examples of MR images available for qualitative analysis. All the images came from the same subject. Cervical levels are located on 3D T2-MERGE and sagittal T2-spin echo. Fusion of FA - 3D MERGE clearly shows that f-FOV DTI and r-FOV 3N/20D are more distorted and more blurred with less anatomic precision than the other r-FOV images.

conducted on a smaller ROI eroded by 2 pixels. The FA values of each ROI were extracted, with right and left measurements being averaged (Fig 1B).

1) Quantifications of residual distortion: The residual distortion after eddy current correction was quantified by a ratio that we called the "distortion ratio" calculated as follows:

Distortion Ratio $=$

$$
\frac{\mid(S(\text { Full Section }- \text { Merge })-S(\text { Full Section }- \text { FA }) \mid}{S(\text { Full Section }- \text { Merge })} \times 100,
$$

where $S$ is the surface of the full section of the cervical spinal cord. The distortion ratio was calculated at the 4 cervical levels and then averaged to obtain 1 single distortion ratio value per patient for each sequence. It ranges from zero when there is no distortion in the FA map compared with the reference anatomic MERGE sequence, and it increases when distortions are more pronounced.

2) Signal-to-noise ratio: Because multichannel coil and parallel imaging were used to collect DTI data, SNR cannot be exactly assessed. ${ }^{18}$ In addition, background noise was not always included within the r-FOV DTI acquisition. Therefore, we estimated spinal cord SNR at B0 and $b=750 \mathrm{~s} / \mathrm{mm}^{2}$ as the ratio between the signal of the full section of the cord and the SD measured within the same neck muscle (longissimus capitis). $\mathrm{SNR}_{\mathrm{B} 0}$ and $\mathrm{SNR}_{\mathrm{b} 750}$ were assessed at the 4 cervical levels and then averaged to obtain single $\mathrm{SNR}_{\mathrm{B} 0}$ and $\mathrm{SNR}_{\mathrm{b} 750}$ values per patient and per DTI sequence.

3) Variability of FA measurements: Because a current issue with CSC DTI is its lack of precision and its variability, even among healthy subjects, we aimed at estimating variations among healthy volunteers. For this purpose, we measured FA on the full section and WM and GM at the 4 levels, and we calculated the mean and SD of these 4 values.

4) Quantitative comparisons based on tractography analysis:
Raw data were postprocessed with Olea Sphere software. Motion correction was performed. We drew 2 seed ROIs that included the entire section of the spine at the $\mathrm{C} 1$ and $\mathrm{C} 3$ levels. We measured the number of fibers detected between these 2 ROIs, per patient and per DTI sequence. The following parameters were used for stopping the tractography: FA minimum $=0.25$, maximum angle $=41.4^{\circ}$, fiber minimum length $=20$ $\mathrm{mm}$.

\section{Statistical Analyses}

All statistical analyses were performed with GraphPad Prism Software, Version 6 (GraphPad Software, San Diego, California). Gaussian distributions were tested with a Shapiro-Wilk normality test. To compare the qualitative scores, distortion ratio, $\mathrm{SNR}_{\mathrm{B} 0}, \mathrm{SNR}_{\mathrm{b} 750}$, mean FA values per topography, and number of detected fibers by tractography, we used 1-way ANOVA (with a post hoc $t$ test) or the Kruskal-Wallis test (with a post hoc Dunn test), depending on the distribution of the variables. For artifact comparisons, contingency tables were built, and we compared sequences with the Fisher exact test to account for the small size of the sample. We performed a Bartlett test to assess whether the FA values of each DTI set had equal variance, after verification of the Gaussian distribution. $P<.05$ was significant.

\section{RESULTS}

No incidental pathologic finding was observed in these young subjects, notably, no degenerative disc disease.

\section{Qualitative Analysis}

Figure 2 shows the superimposition of the FA map with the anatomic reference 3D-T2-MERGE at the 4 levels of interest (C1-C4) and a sagittal reconstruction for the f-FOV and the 5 r-FOV schemes, with the same FA color scale.

On qualitative radiologic assessment, the 5 r-FOV DTI sequences with a NEX of $\geq 5$ were not scored differently from each other but were significantly better evaluated than the f-FOV images $(3 \mathrm{~N} / 20 \mathrm{D})(1.1 \pm 0.8)$ or the r-FOV images (3N/20D) $(1.8 \pm$ $0.56)$. There was no qualitative difference between the f-FOV sequence and the r-FOV 3N/20D sequence (Fig 3).

Specifically, the 2 radiologists consistently observed fewer distortions, less misregistration in the anteroposterior direction, and less blurring for the r-FOV sequences with a NEX of $>5$ than for the 2 other sequences. The r-FOV images obtained with 5N/16D, $7 \mathrm{~N} / 12 \mathrm{D}, 9 \mathrm{~N} / 9 \mathrm{D}$, and 12N/6D provided CSC morphology closer to the anatomic reference as opposed to the $\mathrm{f}-\mathrm{FOV}$ or the $\mathrm{r}-\mathrm{FOV}$ 3N/20D images (Figs 2 and 3).

\section{Number of Sections with Artifacts}

The dataset with the highest number of sections with artifacts was r-FOV 3N/20D (31.6\%) followed by f-FOV (22.7\%), while the 


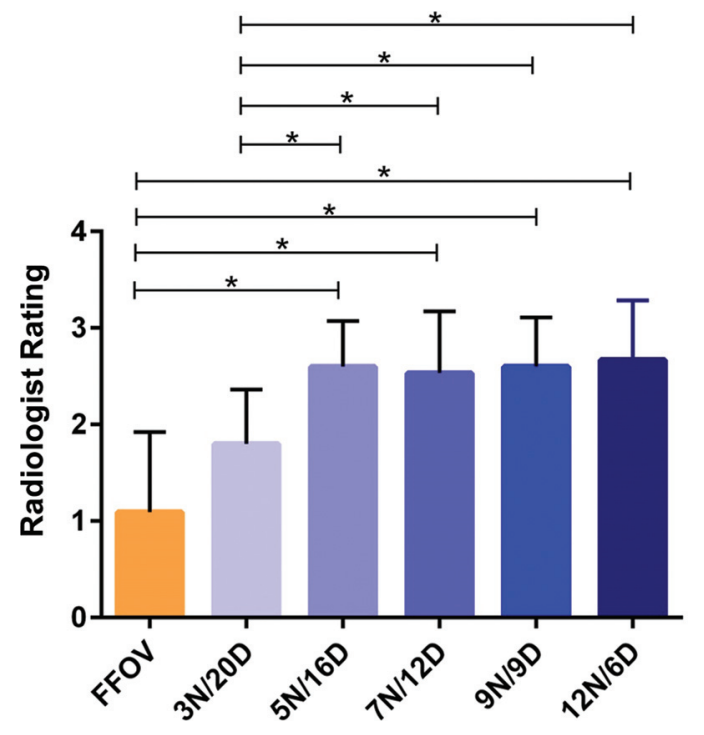

FIG 3. Qualitative analysis. Radiologists determined a rate for each sequence, for each subject, from 1 (nondiagnostic) to 4 (good). Mean rates \pm SDs for the sequence are represented. Superimposed black lines indicate which sequences are statistically different with $P<.05$ (asterisk).

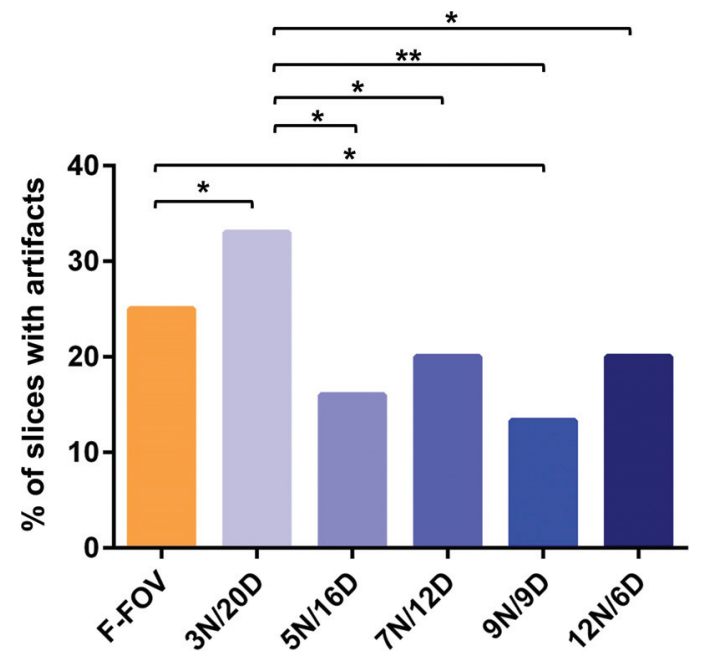

FIG 4. Percentages of sections with artifacts unusable for DTI analysis due to susceptibility artifacts or poor SNR. Superimposed black lines indicate which sequences are statistically different with $P<.05$ (asterisk) and $P<.005$ (double asterisks).

r-FOV sequences with a NEX of $\geq 5$ were significantly better; the r-FOV 9N/9D was the one with fewest artifacts (13.3\%) (Fig 4).

Artifacts were mainly due to CSF flow on B0 images and residual distortions after eddy current correction (the latter being particularly seen on f-FOV and r-FOV 3N/20D), notably at the lower level of the acquisition volume (C4).

\section{Quantitative Comparisons Based on ROI Analysis}

The distortion ratio decreased continuously while increasing the NEX (and, in turn, decreasing the NDGD), even though this effect did not reach statistical significance (Fig $5 A$ ). Thus, the distortion ratios for $\mathrm{r}-\mathrm{FOV} 3 \mathrm{~N} / 20 \mathrm{D}$ and $\mathrm{r}-\mathrm{FOV} 12 \mathrm{~N} / 6 \mathrm{D}$ were $13.14 \pm 6.6 \%$ and $9.25 \pm 5.5 \%$, respectively.

SNR on the B0 map did not show significant differences be- tween the sequences $(P>.05)$. At $b=750 \mathrm{~s} / \mathrm{mm}^{2}$, no significant difference was observed among the DTI sets, except for the comparison between r-FOV 3N/20D and r-FOV 9N/9D, which had, respectively, the lowest and the highest SNR (23.95 \pm 4 versus $36.04 \pm 5.8, P=.0182$ ) (Fig 5B). Considering that a critical threshold of SNR below 8 should lead to section rejection, $10 \%$ of the sections had to be rejected because of a signal below this threshold on $\mathrm{f}-\mathrm{FOV} ; 6.7 \%$, on $\mathrm{r}-\mathrm{FOV} 3 \mathrm{~N} / 20 \mathrm{D}$; and $5 \%$, on $\mathrm{r}-\mathrm{FOV} 7 \mathrm{~N} / 12 \mathrm{D}$ and $\mathrm{r}-\mathrm{FOV} 12 \mathrm{~N} / 6 \mathrm{D}$. The SNR was always above 8 for $\mathrm{r}$-FOV sections acquired with 5N/16D and with 9N/9D.

Whatever the location (full section, GM, and WM) and sequences, comparisons of the distributions of FA values did not reveal a significant difference (Fig $5 C$ ). Furthermore, there was no significant difference between the FA values, depending on the acquisition pulse sequence. For instance, FA values of the full section were the following: $0.70 \pm 0.04$ for $\mathrm{f}-\mathrm{FOV}, 0.74 \pm 0.04$ for r-FOV $3 \mathrm{~N} / 20 \mathrm{D}, 0.71 \pm 0.05$ for $\mathrm{r}-\mathrm{FOV} 5 \mathrm{~N} / 16 \mathrm{D}, 0.7 \pm 0.04$ for r-FOV 7N/12D, $0.074 \pm 0.03$ for r-FOV 9N/9D, and $0.74 \pm 0.04$ for $\mathrm{r}-\mathrm{FOV} 12 \mathrm{~N} / 6 \mathrm{D}$. The FA values that we obtained were consistent with those in the literature, ${ }^{19-22}$ with lower and more dispersed measures within the GM and higher and less dispersed measures within the WM.

\section{Quantitative Comparisons Based on Tractography Analysis}

Figure $6 A$ represents tractograms derived from the 2 seeds superimposed on the corresponding trace at $b=750 \mathrm{~s} / \mathrm{mm}^{2}$. R-FOV $7 \mathrm{~N} / 12 \mathrm{D}, 9 \mathrm{~N} / 9 \mathrm{D}$, and $12 \mathrm{~N} / 6 \mathrm{D}$ qualitatively exhibited a more realistic anatomy of the cord than f-FOV, r-FOV $3 \mathrm{~N} / 20 \mathrm{D}$, and r-FOV $5 \mathrm{~N} / 16 \mathrm{D}$, with more fibers and longer tracts.

Quantitatively, r-FOV 9N/9D detected the highest number of tracts without abnormal tract findings on tractograms (4587 \pm $1743)$, closely followed by r-FOV 12 N/6D (4290 \pm 1206$)$, with findings not significantly different from the previous ones. The best sequence (r-FOV 9N/9D) detected significantly more fibers than f-FOV $(P<.0001)$, r-FOV 5N/16D $(P=.0004)$, and r-FOV 3N/20D $(P<.05)($ Fig $6 B)$.

\section{DISCUSSION}

Our study did not show any significant difference between CSC DTI obtained with f-FOV or r-FOV when using a protocol favoring NDGD over NEX. However, we showed real benefits of the r-FOV by balancing the NDGD and the NEX, the best trade-off being $9 \mathrm{~N} / 9 \mathrm{D}$, in terms of qualitative aspects, reduction of artifacts, SNR, and detection of fibers, while maintaining identical FA values.

This study demonstrates that optimized parameters for CSC DTI in clinical conditions are different from those for brain DTI, which requires a high number of directions, ideally at least 31 while the NEX can be reduced. ${ }^{23}$ Here, we demonstrated that CSC DTI does not require such a high number of gradient directions. In theory, high NDGD leads to more precise DTI information. In the CSC, FA values and their variations were similar with 6, 9, 12, 16 , or 20 NDGD in a set of healthy subjects; this finding might be explained by the strong orientation of the cord in the rostrocaudal axis. However, we recommend increasing the number of averages 

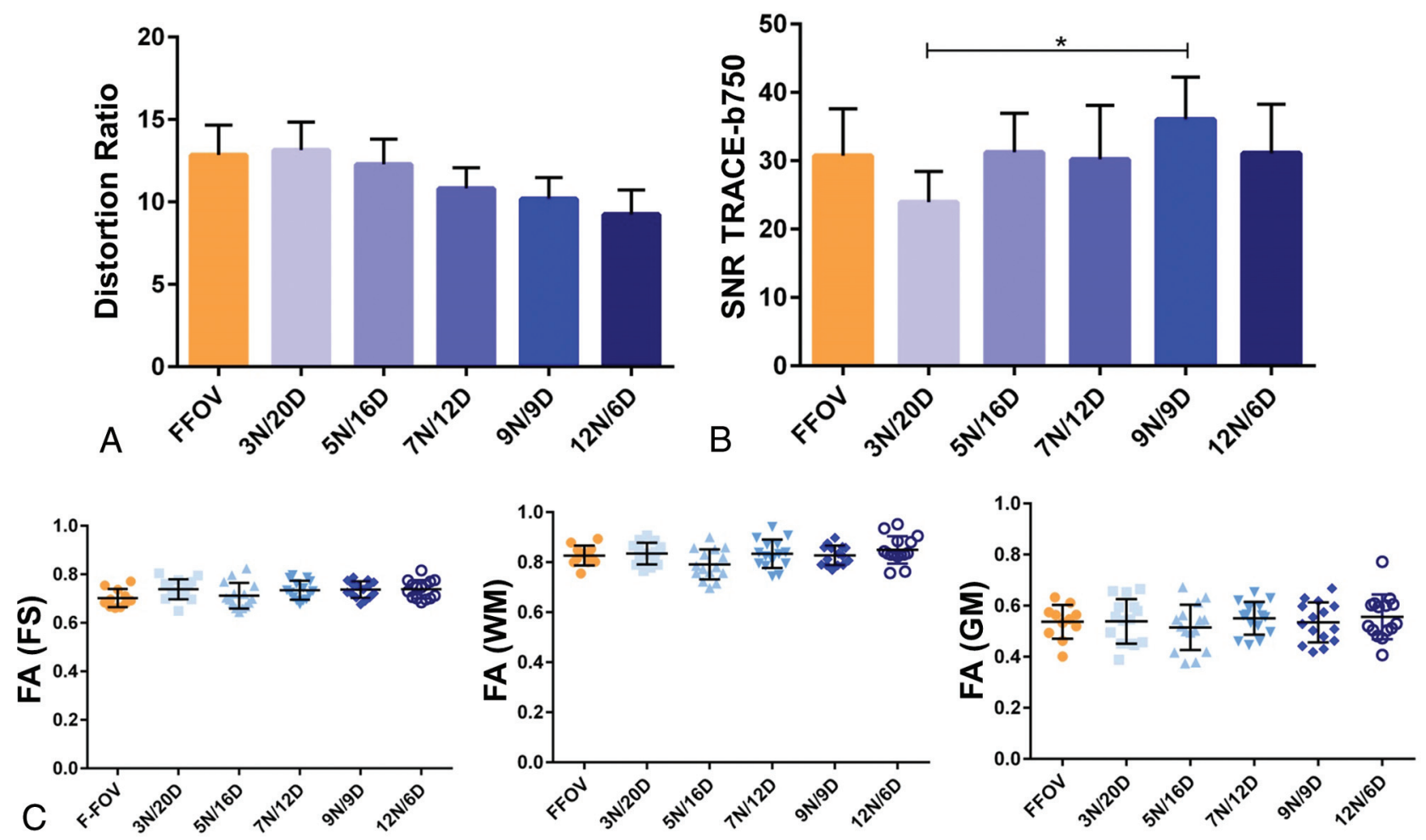

FIG 5. Quantitative comparisons on ROI-based analyses. A, The distortion ratio. $B$, The SNR on the trace image at $b=750 \mathrm{~s} / \mathrm{mm}^{2}$. C, Representation of the dispersion of FA values, depending on the DTI sequence and, successively, a full section of the spinal cord (FS), WM, and GM. Mean rates \pm SDs for the sequence are represented. Superimposed black lines indicate which sequences are statistically different with $P<.05$ (asterisk).

because the CSC has low intrinsic signal and is prone to artifacts that can be averaged and canceled (such as flow artifacts) with several excitations. Altogether, we recommend choosing NEX over NDGD when time is limited as opposed to standard recommendation for brain exploration. These results might be particularly important when axial sections are used because the axial section is prone to flow artifacts. Other strategies could be adopted and associated in the future to further limit the flow artifacts: 1) Many B0s could be acquired to select the one with the fewest artifacts; 2) cardiac gating could be added but at the cost of increasing scan time; 3) 2 B0 acquisitions could be averaged, in phase and in reversed phase; and 4) the tensor could be estimated from the $b=50 \mathrm{~s} / \mathrm{mm}^{2}$ and $b=750 \mathrm{~s} / \mathrm{mm}^{2}$ acquisition without requiring $\mathrm{B} 0$.

In line with this conclusion, previous studies dealing with the optimization of DTI parameters for CSC have also demonstrated, in a similar sequential approach, that there was no need to drastically increase NDGD (15 was satisfying compared with 32), while the NEX had to be increased. ${ }^{13}$ However, these results were obtained with a f-FOV sequence, and their relevance might be still more important with a r-FOV strategy.

Other kinds of r-FOV sequences exist, depending on the MR imaging system, but all of them rely on the same principle. Reducing the excited region in the phase-encoding direction enables a shorter echo train, increased blip moment, and, thus, reduced susceptibility effects. ${ }^{24}$ Spatial definition can be improved. SNR and scan time are supposed to remain stable. Among the r-FOV methods, the most popular are the following: 1) ZOnal Oblique Multislice-EPI, ${ }^{11}$ an inner volume technique, in which a refocus- ing pulse in an orthogonal or oblique plane to the excitation plane is applied, but with the creation of section gaps; 2) inner volume excitation and outer volume suppression ${ }^{10}$; and 3 ) the r-FOV. ${ }^{6}$ As a consequence of the increased image quality, applications of $\mathrm{r}$ FOV sequences are increasingly used not only for the CSC but also to explore the midbrain, optic nerves, or hippocampi for neuroradiology, and also the pancreas and prostate. ${ }^{25,26}$

Currently, there is no validated method for CSC DTI quality control. The CSC and its environment are too complex for mathematic $^{15}$ or phantom simulations. The method that we proposed here can be viewed as a realistic bench test that can be performed by any radiologist and that covers all aspects of quality control.

Finally, as additional techniques are being developed, we can expect further improvement: 1) The combination of parallel imaging and r-FOV, which has already been performed for the pons at $7 \mathrm{~T}^{27}$, can still increase DTI quality; 1) multisegmented 3D-EPI could increase the SNR and could be associated with r-FOV and parallel imaging to reduce the echo $\operatorname{train}^{28} ; 3$ ) a more accurate diffusion model could be used such as neurite orientation dispersion and density imaging, ${ }^{29}$ which might provide more accurate quantitative diffusion metrics, especially for anisotropic structures like gray matter; and 4) improved postprocessing methods are being developed, ${ }^{30}$ with improved eddy current and motion corrections.

Our study has limitations. We did not investigate other important parameters such as b-value, section thickness, bandwidth, cardiac gating, respiratory gating, and different designs of diffusion vectors. However, these parameters were set regardless of the 

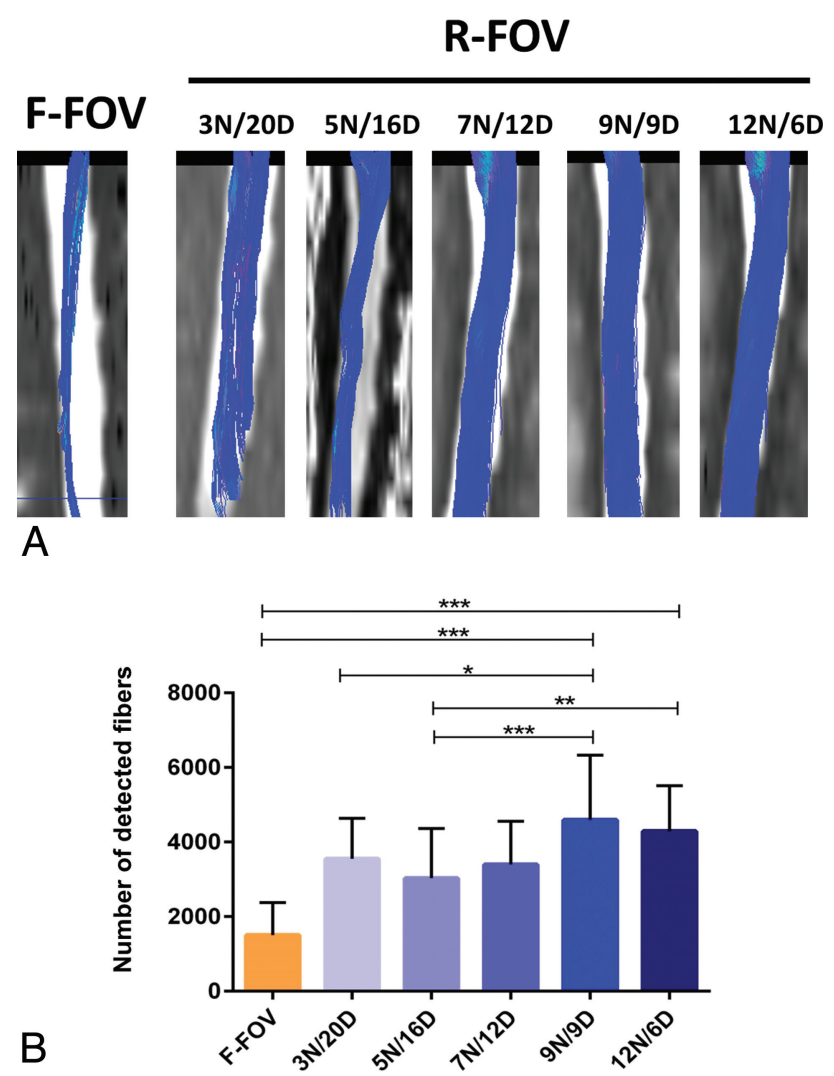

FIG 6. Tractography-based analyses. A, The reconstructed tractograms for the whole DTI dataset. For each DTI sequence, 2 similar seed ROls were placed on the anatomic sequence, at the $\mathrm{Cl}$ and $\mathrm{C} 3$ levels, and then propagated on diffusion data. Care was taken to exclude abnormal fiber detection (ie, in the CSF). Qualitatively, r-FOV sequences clearly exhibit better tractogram definitions. $B$, The number of detected fibers between the 2 seeds. $P<.05$ (asterisk), $P<.005$ (double asterisks), $P<.0005$ (triple asterisks).

sequence. The values we used ranged among those classically recommended ${ }^{31}$ notably by MR imaging constructors. Furthermore, all our acquisitions were performed on the same $3 \mathrm{~T} \mathrm{MR}$ imaging system. Further studies are required to investigate whether our results could be translated to $1.5 \mathrm{~T}$. The current literature suggests that $1.5 \mathrm{~T}$ and $3 \mathrm{~T}$ should provide similar DTI results, though a direct comparison has not been conducted for CSC DTI, to our knowledge. Phantom studies with classic diffusion have shown that the increased SNR inherent at higher magnetic fields was counterbalanced by increased distortions or susceptibility artifacts. ${ }^{32}$ For brain DTI, Grech-Sollars et a ${ }^{33}$ recently concluded that no significant difference was observed in the interscanner coefficient of variation for mean diffusivity and FA when $1.5 \mathrm{~T}$ and $3 \mathrm{~T}$ systems were compared with similar brain DTI protocols. Therefore, the parameter adjustments that we suggest at $3 \mathrm{~T}$ might translate to $1.5 \mathrm{~T}$, but a formal comparison will be needed to validate this statement. In addition, our patient population consisted of only healthy young adults without significant medical histories, which might have led to better quality than what can be encountered in clinical routine. Degenerative, inflammatory, traumatic, metabolic, or tumoral spinal diseases can modify diffusivity parameters. ${ }^{1-5}$ Consequently, even though our study design compared the DTI sequences, the values of our judgment criteria cannot be translated directly into clinical practice.
Another limitation is that we studied only 15 healthy subjects, which might not provide the statistical power to detect subtle differences. Nonetheless, this sample already reveals a better set of parameters, namely r-FOV 9N/9D.

\section{CONCLUSIONS}

R-FOV CSC DTI is clinically feasible and provides significant qualitative and quantitative improvement when optimized. Contrary to brain imaging, we recommend limiting NDGD and increasing NEX because the CSC is a small highly oriented structure generating low signal and is subject to artifacts. The best compromise in a clinically acceptable scan time of 4 minutes 30 seconds is r-FOV with a NEX of 9 and 9 NDGD.

\section{ACKNOWLEDGMENTS}

We thank Gerard Raffard for his help with image analyses in this study.

Disclosures: Thomas Tourdias-UNRELATED: Grants/Grants Pending: ANR-10LABX-57 named TRAIL and ANR-10-LABX-43 named BRAIN. * Marion Uettwiller-I am a General Electric Healthcare employee working as an application specialist. The system used for this study was a General Electric system. I assisted in establishing the acquisition protocol and provided technical information included in the article. *Money paid to the institution.

\section{REFERENCES}

1. Shanmuganathan K, Gullapalli R, Zhuo J, et al. Diffusion tensor MR imaging in cervical spine trauma. AJNR Am J Neuroradiol 2008;29: 655-59 CrossRef Medline

2. Facon D, Ozanne A, Fillard P, et al. MR diffusion tensor imaging and fiber tracking in spinal cord compression. AJNR Am J Neuroradiol 2005;26:1587-94 Medline

3. Renoux J, Facon D, Fillard P, et al. MR diffusion tensor imaging and fiber tracking in inflammatory diseases of the spinal cord. AJNR Am J Neuroradiol 2006;27:1947-51 Medline

4. van Hecke W, Nagels G, Emonds G, et al. A diffusion tensor imaging group study of the spinal cord in multiple sclerosis patients with and without T2 spinal cord lesions. J Magn Reson Imaging 2009;30: 25-34 CrossRef Medline

5. Budzik JF, Balbi V, Le Thuc V, et al. Diffusion tensor imaging and fibre tracking in cervical spondylotic myelopathy. Eur Radiol 2011; 21:426-33 CrossRef Medline

6. Saritas EU, Cunningham CH, Lee JH, et al. DWI of the spinal cord with reduced FOV single-shot EPI. Magn Reson Med 2008;60: 468-73 CrossRef Medline

7. Kim TH, Zollinger L, Shi XF, et al. Quantification of diffusivities of the human cervical spinal cord using a 2D single-shot interleaved multisection inner volume diffusion-weighted echo-planar imaging technique. AJNR Am J Neuroradiol 2010;31:682-87 CrossRef Medline

8. Finsterbusch J. High-resolution diffusion tensor imaging with inner field-of-view EPI. J Magn Reson Imaging 2009;29:987-93 CrossRef Medline

9. Jeong EK, Kim SE, Guo J, et al. High-resolution DTI with 2D interleaved multislice reduced FOV single-shot diffusion-weighted EPI (2D ss-rFOV-DWEPI). Magn Reson Med 2005;54:1575-79 CrossRef Medline

10. Wilm BJ, Svensson J, Henning A, et al. Reduced field-of-view MRI using outer volume suppression for spinal cord diffusion imaging. Magn Reson Med 2007;57:625-30 CrossRef Medline

11. Wheeler-Kingshott CA, Hickman SJ, Parker GJ, et al. Investigating cervical spinal cord structure using axial diffusion tensor imaging. Neuroimage 2002;16:93-102 CrossRef Medline

12. Zaharchuk G, Saritas EU, Andre JB, et al. Reduced field-of-view diffusion imaging of the human spinal cord: comparison with conven- 
tional single-shot echo-planar imaging. AJNR Am J Neuroradiol 2011;32:813-20 CrossRef Medline

13. Lee JW, Kim JH, Kang HS, et al. Optimization of acquisition parameters of diffusion-tensor magnetic resonance imaging in the spinal cord. Invest Radiol 2006;41:553-59 CrossRef Medline

14. Crombe A, Menegon P, Tourdias T, et al. Diffusion tensor imaging of cervical spinal cord at $3 \mathrm{~T}$ : normal values of radial, axial, mean diffusivities and fractional anisotropy in anterior horn, dorsal funiculus and pyramidal tract at each cervical level. In: Proceedings of the International Society for Magnetic Resonance in Medicine and the European Society for Magnetic Resonance in Medicine Joint Annual Meeting, Milan, Italy. May 10-16, 2014

15. Gao W, Zhu H, Lin W. A unified optimization approach for diffusion tensor imaging technique. Neuroimage 2009;44:729-41 Medline

16. Mukherjee P, Chung SW, Berman JL, et al. Diffusion tensor MR imaging and fiber tractography: technical considerations. AJNR Am J Neuroradiol 2008;29:843-52 CrossRef Medline

17. Jones DK, Horsfield MA, Simmons A. Optimal strategies for measuring diffusion in anisotropic systems by magnetic resonance imaging. Magn Reson Med 1999;42:515-25 Medline

18. Dietrich O, Raya JG, Reeder SB, et al. Measurement of signal-tonoise ratios in MR images: influence of multichannel coils, parallel imaging, and reconstruction filters. J Magn Reson Imaging 2007;26: 375-85 CrossRef Medline

19. Brander A, Koskinen E, Luoto TM, et al. Diffusion tensor imaging of the cervical spinal cord in healthy adult population: normative values and measurement reproducibility at 3T MRI. Acta Radiol 2014; 55:478-85 CrossRef Medline

20. Xu J, Shimony JS, Klawiter EC, et al. Improved in vivo diffusion tensor imaging of human cervical spinal cord. Neuroimage 2013;67: 64-76 CrossRef Medline

21. Tang L, Wen $Y$, Zhou Z, et al. Reduced field-of-view DTI segmentation of cervical spine tissue. Magn Reson Imaging 2013;31:1507-14 CrossRef Medline

22. Ellingson B, Ulmer JL, Kurpad SN, et al. Diffusion tensor MR imag- ing of the neurologically intact human spinal cord. AJNR Am J Neuroradiol 2008;29:1279-84 CrossRef Medline

23. $\mathrm{Ni} \mathrm{H}$, Kavcic $\mathrm{V}$, Zhu T, et al. Effects of number of diffusion gradient directions on derived diffusion tensor imaging indices in human brain. AJNR Am J Neuroradiol 2006;27:1776-81 Medline

24. Viallon M, Cuvinciuc V, Delattre B, et al. State-of-the-art MRI techniques in neuroradiology: principles, pitfalls, and clinical applications. Neuroradiology 2015;57:441-67 CrossRef Medline

25. Ma C, Li YJ, Pan CS, et al. High resolution diffusion weighted magnetic resonance imaging of the pancreas using reduced field of view single-shot echo-planar imaging at 3 T. Magn Reson Imaging 2014; 32:125-31 CrossRef Medline

26. Korn N, Kurhanewicz J, Banerjee S, et al. Reduced-FOV excitation decreases susceptibility artifact in diffusion-weighted MRI with endorectal coil for prostate cancer detection. Magn Reson Imaging 2015;33:56-62 CrossRef Medline

27. Wargo CJ, Gore JC. Localized high-resolution DTI of the human midbrain using single-shot EPI, parallel imaging, and outer-volume suppression at 7T. Magn Reson Imaging 2013;31:810-19 CrossRef Medline

28. Engström M, Skare S. Diffusion-weighted 3D multislab echo planar imaging for high signal-to-noise ratio efficiency and isotropic image resolution. Magn Reson Med 2013;70:1507-14 CrossRef Medline

29. Grussu F, Schneider T, Zhang H, et al. Neurite orientation dispersion and density imaging of the healthy cervical spinal cord in vivo. Neuroimage 2015;111:590-601 CrossRef Medline

30. Mohammadi S, Freund P, Feiweier T, et al. The impact of post-processing on spinal cord diffusion tensor imaging. Neuroimage 2013; 70:377-85 CrossRef Medline

31. Jones D. Diffusion MRI: Theory, Methods, and Applications. New York: Oxford University Press; 2011

32. Lavdas I, Miquel ME, McRobbie DW, et al. Comparison between diffusion-weighted MRI (DW-MRI) at 1.5 and 3 Tesla: a phantom study. J Magn Reson Imaging 2014;40:682-90 CrossRef Medline

33. Grech-Sollars M, Hales PW, Miyazaki K, et al. Multi-centre reproducibility of diffusion MRI parameters for clinical sequences in the brain. NMR Biomed 2015;28:468-85 CrossRef Medline 